\title{
Associations between Phase Angle Values Obtained by Bioelectrical Impedance Analysis and Nonalcoholic Fatty Liver Disease in an Overweight Population
}

\author{
Guangya Chen, ${ }^{1}$ Yi Lv, ${ }^{2,3}$ Wei Ni, ${ }^{2,3}$ Qingxin Shi, ${ }^{1}$ Xingliang Xiang, ${ }^{1}$ Sen Li, ${ }^{4}$ Chengwu Song, \\ Mingzhong Xiao $\mathbb{D},{ }^{2,3}$ and Shuna Jin $\mathbb{1}^{5}$ \\ ${ }^{1}$ College of Pharmacy, Hubei University of Chinese Medicine, Wuhan, Hubei, China \\ ${ }^{2}$ Hepatic Disease Institute, Hubei Provincial Hospital of Traditional Chinese Medicine, Wuhan, Hubei, China \\ ${ }^{3}$ Hubei Provincial Academy of Traditional Chinese Medicine, Wuhan, Hubei, China \\ ${ }^{4}$ Department of Pharmacy, Union Hospital, Tongji Medical College, Huazhong University of Science and Technology, Wuhan, \\ Hubei, China \\ ${ }^{5}$ Key Laboratory of Environment and Health, Ministry of Education and Ministry of Environmental Protection, \\ State Key Laboratory of Environmental Health, School of Public Health, Tongji Medical College, \\ Huazhong University of Science and Technology, Wuhan, Hubei, China
}

Correspondence should be addressed to Mingzhong Xiao; xmz0001@sohu.com and Shuna Jin; jinshuna@hust.edu.cn

Received 7 May 2020; Revised 22 June 2020; Accepted 25 June 2020; Published 5 August 2020

Academic Editor: Alessandro Granito

Copyright $(2020$ Guangya Chen et al. This is an open access article distributed under the Creative Commons Attribution License, which permits unrestricted use, distribution, and reproduction in any medium, provided the original work is properly cited.

Objective. There is a limited diagnosis of nonalcoholic fatty liver disease (NAFLD). Thus, the noninvasive assessments are worth exploring. We determined the associations of phase angles ( $\mathrm{PhAs}$ ) obtained from bioelectric impedance analysis (BIA) with the risk of NAFLD in an overweight population. Methods. A study involving 953 overweight participants was conducted in Wuhan city, China. The associations between PhAs (right arm, left arm, body trunk, right leg, left leg, and whole body) and the risk of NAFLD were conducted using multivariate logistic regression analyses. The associations of PhAs with the controlled attenuation parameter (CAP), a noninvasive assessment of liver steatosis and fibrosis, were also evaluated by both linear and logistic regression analyses. Results. The PhA values of the whole body, trunk, and legs were significantly lower $(P<0.05)$ in the NAFLD group than the non-NAFLD group. After adjustment for BMI, gender, education, income/year, hyperlipidemia, hypertension, diabetes, smoking, passive smoking, and drinking, significant associations of PhA values of the right leg, left leg, and whole body with the risk of NAFLD were observed. In addition, the PhA of the right leg, left leg, and whole body were significantly related to the CAP values. Further stratified analyses indicated that these associations were significant in the participants with $\mathrm{BMI}<30$, but not in the participants with $\mathrm{BMI} \geq 30$. Conclusions. PhAs might be effective indicators in the management of NAFLD among overweight people.

\section{Introduction}

Nonalcoholic fatty liver disease (NAFLD) is a clinicopathological liver disease, which is characterized by macrovesicular hepatic lipids accumulation and the occurrence in patients who consume little or even no alcohol. NAFLD is not a single disease and usually can be divided into three stages: nonalcoholic fatty liver, nonalcoholic steatohepatitis, and liver cirrhosis [1]. With the increase of the obesity epidemic throughout the world, NAFLD is one of the most common liver diseases both in the developed and developing world with an estimated prevalence of $20-40 \%$ [2]. Although the exact prevalence varies from region to region, the overall trend shows an increased number of patients with NAFLD. In the North of the Netherlands, $22 \%$ of adults suffered from NAFLD [3]. A recent report in Beijing indicated that the prevalence of NAFLD in adults was $31.0 \%$ in the Asiatic population [4]. NAFLD has rapidly become a leading 
indication of liver transplantation because of its increasing prevalence and lack of effective therapies [5].

Until now, the pathogenesis of NAFLD is not fully clear. High-fat diets and physical inactivity are strongly associated with the development and progression of NAFLD [6]. In addition, there was evidence that increased body fat, particularly abdominal visceral fat, was central in the pathogenesis of NAFLD [7, 8]. However, it is a fact that not all overweight people have NAFLD. Therefore, it is critical to explore early diagnosis methods or indicators to prevent the occurrence of NAFLD in the high incidence population, such as overweight or obese people.

The diagnosis of NAFLD requires imaging or histological evidence of an excessive accumulation of triglyceride in hepatocytes. The gold standard for the diagnosis remains the percutaneous liver biopsy. However, because of increased cost, higher risk, and health care resource use, invasive liver biopsy is a poorly suited diagnostic test for such a prevalent disease [9]. Bioelectrical impedance analysis (BIA) is a noninvasive tool for assessing body composition [10]. It measures the resistance and capacitance of body components by recording a voltage drop in the applied current [11]. Capacitance causes the current to lag behind the voltage, resulting in a phase shift. This shift is quantified geometrically as the angular transformation-the phase angle (PhA). Previous reports showed that the PhA was strongly associated with a poor outcome in clinical conditions. Gupta et al. demonstrated that PhA was a strong predictor of survival in breast cancer [12]. Two cross-sectional studies in Germany indicated that higher PhA was associated with lower relative mortality risk [13]. A case-control study including 983 healthy adults and 983 admitted patients suggested a significant association between low PhA and nutritional risk [14]. However, it remains unclear whether $\mathrm{PhA}$ can be a predictor for the risk of NAFLD in overweight people.

Therefore, we sought to determine the associations of NAFLD with PhAs of the whole body, trunk, arms, and legs obtained from BIA in an overweight population. In addition, the associations between PhAs and the controlled attenuation parameter (CAP), a noninvasive assessment of liver steatosis and fibrosis, were also investigated.

\section{Materials and Methods}

2.1. Study Subject. This study recruited 953 overweight participants at Hubei Provincial Hospital of Traditional Chinese Medicine in 2016. The overweight was defined as a body mass index $(\mathrm{BMI}) \geq 24.0 \mathrm{~kg} / \mathrm{m}^{2}$ according to the Chinese reference standard. The participants met the following criteria: (1) $\mathrm{BMI} \geq 24.0 \mathrm{~kg} / \mathrm{m}^{2}$, (2) aged $18-60$, (3) written informed consent, and (4) ability to comprehend the Chinese language and complete the questionnaire. Besides, all participants provided written informed consent at enrollment, and the ethics protocol was acquired by the Ethics Committee of Hubei Provincial Hospital of Traditional Chinese Medicine.
2.2. Data Collection. We used structured and validated questionnaires to obtain information on socioeconomic and lifestyle characteristics, such as age, education, income/year, frequency of alcohol intake (how many times per week), and smoking status. Height and body weight were measured in participants wearing light clothing without shoes. BMI was calculated as body weight in kilograms divided by the square of height $\left(\mathrm{kg} / \mathrm{m}^{2}\right)$. Hypertension was defined as Diastolic Blood Pressure $\geq 90 \mathrm{mmHg}$ and (or) Systolic Blood Pressure $\geq 140 \mathrm{mmHg}$ at examinations or having hypertension history. Diabetes was defined as fasting plasma glucose $\geq 7.0 \mathrm{mmol} / \mathrm{L}$ or having diabetes history.

2.3. PhA Determination. PhA was assessed by a bioelectric impedance device (InBdoy 770, InBody, China). The test procedure was conducted according to the manufacturer's instructions. Participants removed all metal objects and other items that might interfere with the scan and emptied the bladder. Participants stepped on the multifrequency BIA device with bare feet, held hands on the hand rails, and stayed on the device for two minutes. The analyzer uses an alternate current of $80 \mathrm{~mA}$ and assesses the $\mathrm{PhA}$ at $50 \mathrm{kHz}$. The MF-BIA device measures segmental impedances at the right arm, left arm, right leg, left leg, and trunk for six frequencies. The PhA for each half of the body at each frequency is then calculated using the following formula: $\mathrm{PhA}=\arctan (\mathrm{Xc} / R) \times 180 / \pi(\mathrm{Xc}$ is the reactance and $R$ is the body resistance).

2.4. NAFLD Diagnosis and CAP Determination. The diagnosis of NAFLD in this study was determined by abdominal echography with the characteristic of "bright liver" and evidence of hepatic and renal parenchyma, vessel blurring, focal sparing, and hepatic veins stenosis, according to the Chinese Guidelines for the Diagnosis and Management of Nonalcoholic Fatty Liver Disease (update in 2010) [15]. Ultrasonography was performed by experienced radiologists to diagnose NAFLD.

Currently, because CAP has been reported to perform well for mild steatosis on people with NAFLD [16], the normal range was less than $240 \mathrm{~dB} / \mathrm{min}$ and our study also tested CAP values. CAP was measured by the ultrasonic attenuation at $3.5 \mathrm{MHz}$ using the FibroScan (EchoSens, Paris, France). CAP was developed on the postulate that fat affected ultrasonic propagation. The value was based on the degree of ultrasonic attenuation by hepatic fat at the central frequency of the FibroScan ${ }^{\circledR} \mathrm{M}$ probe simultaneously with liver stiffness measurement [17].

2.5. Statistical Analysis. All statistical analyses were performed using SAS (version 9.4; SAS Institute Inc.). Data were expressed in means with standard deviations (SD) and odds ratio (OR) with a $95 \%$ confidence interval (CI). The $\mathrm{PhA}$ values at a frequency of $50 \mathrm{kHz}$ in NAFLD and control people were compared by independent sample $t$-tests. The associations between NAFLD and PhAs (right arm; left arm; body trunk; right leg; left leg; whole body) were estimated 
TABle 1: Baseline characteristics $(N=953)$.

\begin{tabular}{|c|c|}
\hline Characteristics & Mean \pm SD or $N(\%)$ \\
\hline Age (years) & $44.0 \pm 9.7$ \\
\hline$\leq 40$ & $325(34.1 \%)$ \\
\hline $40-51$ & $318(33.4 \%)$ \\
\hline$>51$ & $310(32.5 \%)$ \\
\hline BMI $\left(\mathrm{kg} / \mathrm{m}^{2}\right)$ & $28.4 \pm 3.1$ \\
\hline $24.0-26.9$ & $372(39.0 \%)$ \\
\hline $27.0-29.9$ & $345(36.0 \%)$ \\
\hline$\geq 30$ & $235(24.7 \%)$ \\
\hline Missing & $1(0.1 \%)$ \\
\hline \multicolumn{2}{|l|}{ Gender } \\
\hline Male & $325(34.1 \%)$ \\
\hline Female & $628(65.9 \%)$ \\
\hline \multicolumn{2}{|l|}{ Education } \\
\hline Less than high school & $292(30.6 \%)$ \\
\hline High school & $370(38.8 \%)$ \\
\hline More than high school & $291(30.5 \%)$ \\
\hline \multicolumn{2}{|l|}{ Income/year (yuan) } \\
\hline$<50000$ & $317(33.3 \%)$ \\
\hline $50000-100000$ & $453(47.5 \%)$ \\
\hline$>100000$ & $182(19.1 \%)$ \\
\hline Missing & $1(0.1 \%)$ \\
\hline \multicolumn{2}{|l|}{ Fatty liver } \\
\hline Yes & $271(28.4 \%)$ \\
\hline No & $682(71.6 \%)$ \\
\hline \multicolumn{2}{|l|}{ Hyperlipidemia } \\
\hline Yes & $123(12.9 \%)$ \\
\hline No & $830(87.1 \%)$ \\
\hline \multicolumn{2}{|l|}{ Hypertension } \\
\hline Yes & $182(19.1 \%)$ \\
\hline No & $771(80.9 \%)$ \\
\hline \multicolumn{2}{|l|}{ Diabetes } \\
\hline Yes & $51(5.4 \%)$ \\
\hline No & $902(94.6 \%)$ \\
\hline \multicolumn{2}{|l|}{ Smoking } \\
\hline Yes & $187(19.6 \%)$ \\
\hline No & $765(80.3 \%)$ \\
\hline Missing & $1(0.1 \%)$ \\
\hline \multicolumn{2}{|l|}{ Passive smoking } \\
\hline Yes & $616(64.6 \%)$ \\
\hline No & $332(34.8 \%)$ \\
\hline Missing & $5(0.5 \%)$ \\
\hline \multicolumn{2}{|l|}{ Drinking } \\
\hline Yes & $149(15.6 \%)$ \\
\hline No & $804(84.4 \%)$ \\
\hline
\end{tabular}

using logistic regression models. The relationships between $\mathrm{CAP}$ and PhAs (right arm; left arm; body trunk; right leg; left leg; whole body) were explored using linear regression models. Logistic regression analyses were also used to estimate the associations between CAP and PhAs with a cutoff at $240 \mathrm{~dB} / \mathrm{min}$ for CAP. Besides, we also conducted stratified analyses by BMI (BMI <30 and BMI $\geq 30)$ to explore the differences in the associations of PhA with NAFLD and CAP among different BMI groups. Covariates were selected as potential confounders because they were reported to be associated with NAFLD or the $P$-value $<0.1$ on the basis of the statistical consideration. Age, BMI, gender, hyperlipidemia hypertension, diabetes, smoking, passive smoking, drinking, education, and income were adjusted in the final models [18-21]. Two-sided $P$-values of $<0.05$ were considered statistically significant.

\section{Results}

Table 1 lists the characteristics of 953 participants with BIA assessment. There were 628 female and 325 male participants in our study. The mean age was $44.0 \pm 9.7$ years. The BMI in $24.0-26.9 \mathrm{~kg} / \mathrm{m}^{2}$ was $39.0 \%$, in $27.0-29.9 \mathrm{~kg} / \mathrm{m}^{2}$ was $36.2 \%$. The participants of less than high school were $30.6 \%$ and more than high school were $30.5 \%$. About $33.3 \%$ of people had a family income of less than 50000 yuan per year. There were $15.6 \%$ of participants with drinking, $19.6 \%$ with smoking, and $64.6 \%$ with passive smoking. In our study population, $12.9 \%, 19.10 \%$, and $5.35 \%$ of participants had suffered from hyperlipidemia, hypertension, and diabetes, respectively. In addition, there were 271 non-NAFLD and 682 NAFLD participants in our population.

The levels of PhA in different body parts and CAP are shown in Table 2. The results indicated that the trunk PhA had the highest mean level and trunk PhA levels in the NAFLD group were significantly higher than the nonNAFLD group $(P=0.039)$. Besides, PhA levels of the right leg, left leg, and whole body in the NAFLD group were also higher than the non-NAFLD group (all $P<0.05$ ). However, the PhA levels of both the right and left arms had no differences between the NAFLD and non-NAFLD groups. Meanwhile, the CAP levels in the NAFLD group were differed by levels in the non-NAFLD group and the participants with NAFLD disease had higher CAP levels $(P<0.001)$.

Table 3 displays the relationships of NAFLD with PhAs and the characteristics of study participants. We found that participants' age, BMI, hyperlipidemia, hypertension, and diabetes were associated with NAFLD in univariate analysis (all $P<0.01$ ). When the age, BMI, gender, education, income/year, hyperlipidemia, hypertension, diabetes, smoking, passive smoking, drinking, and the PhA were included in the multivariate model, there were still significant associations of NAFLD with the participants' age, BMI, and hyperlipidemia. Besides, the relationships of PhAs in six body parts (right arm, left arm, trunk, right leg, left leg, and whole body) with the risk of NAFLD were further investigated. Our results showed that PhAs of the trunk, right leg, left leg, and whole body were associated with the risk of NAFLD in univariate analysis (all $P<0.05$ ). When in multivariate models, significant associations were found in the PhAs of the right leg, left leg and whole body (for right leg, $\mathrm{OR}=1.41,95 \% \mathrm{CI}: 1.08$ to 1.83 ; for left leg, $\mathrm{OR}=1.30$, 95\% CI: 0.01 to 1.66 ; for whole body, $1.40,95 \%$ CI: 1.03 to 1.91; all $P<0.05)$.

The associations between CAP and PhAs at frequency of $50 \mathrm{kHz}$ are shown in Table 4 . We conducted the univariate and multivariate linear regression between CAP and PhAs of six body parts. In multivariate analyses, the $\mathrm{PhAs}$ of the right leg, left leg, and whole body showed significantly linear associations with CAP values (for right leg, $\beta=9.69,95 \% \mathrm{CI}$ : 4.79 to 14.59 ; for right leg, $\beta=9.40,95 \% \mathrm{CI}: 4.33$ to 14.46 ; for whole body, $\beta=7.20,95 \% \mathrm{CI}$ : 1.32 to 13.09 ; all $P<0.05$ ). 
TABle 2: Phase angles at a frequency of $50 \mathrm{kHz}$ in the study participants.

\begin{tabular}{lccc}
\hline & Total $(N=953)$ & Non-NAFLD $(N=271)$ & NAFLD $(N=682)$ \\
\hline Right arm & $5.10 \pm 0.64$ & $5.07 \pm 0.60$ & $5.11 \pm 0.66$ \\
Left arm & $4.94 \pm 0.64$ & $4.90 \pm 0.60$ & $4.96 \pm 0.66$ \\
Trunk & $7.20 \pm 1.14$ & $7.09 \pm 1.08$ & $7.26 \pm 1.12$ \\
Right leg & $5.86 \pm 0.75$ & $5.76 \pm 0.73$ & $5.90 \pm 0.76$ \\
Left leg & $5.81 \pm 0.74$ & $5.72 \pm 0.71$ & $5.84 \pm 0.75$ \\
Whole body & $5.50 \pm 0.65$ & $5.43 \pm 0.60$ & $5.53 \pm 0.66$ \\
CAP & $251.58 \pm 47.86$ & $221.98 \pm 45.74$ & $263.24 \pm 43.48$ \\
\hline
\end{tabular}

All $P$ values were calculated using independent sample $t$-tests. CAP: controlled attenuation parameter. $P$ values of $<0.05$ were considered statistically significant.

TABLE 3: Univariate and multivariate logistic regression analyses between phase angles and NAFLD.

\begin{tabular}{lccc}
\hline Characteristics & \multicolumn{1}{c}{ Univariate analysis } & Multivariate analyses \\
& OR $(95 \% \mathrm{CI})$ & $P$ value & OR $(95 \% \mathrm{CI})$ \\
\hline Age & $1.02(1.01$ to 1.03$)$ & $<\mathbf{0 . 0 1}$ & $1.03(1.01$ to 1.05$)$ \\
BMI & $3.36(2.68$ to 4.22$)$ & $<\mathbf{0 . 0 1}$ & $3.21(2.54$ to 4.06$)$ \\
Gender & $0.82(0.61$ to 1.11$)$ & 0.20 & $0.96(0.60$ to 1.53$)$ \\
Education & $0.97(0.81$ to 1.16$)$ & 0.76 & $1.19(0.94$ to 1.50$)$ \\
Income/year & $1.03(0.84$ to 1.25$)$ & 0.79 & $1.01(0.80$ to 1.27$)$ \\
Hyperlipidemia & $4.20(2.28$ to 7.76$)$ & $<\mathbf{0 . 0 1}$ & $3.08(1.59$ to 5.97$)$ \\
Hypertension & $2.20(1.45$ to 3.34$)$ & $<\mathbf{0 . 0 1}$ & $1.26(0.78$ to 2.03$)$ \\
Diabetes & $3.84(1.51$ to 9.78$)$ & $<.05(0.75$ to 5.58$)$ \\
Smoking & $1.23(0.85$ to 1.77$)$ & 0.28 & $1.14(0.70$ to 1.86$)$ \\
Passive smoking & $1.00(0.75$ to 1.35$)$ & 0.99 & $0.94(0.68$ to 1.31$)$ \\
Drinking & $0.81(0.55$ to 1.18$)$ & 0.27 & $0.71(0.44$ to 1.13$)$ \\
Phase angles & & & \\
Right arm & & 0.36 & $1.15(0.84$ to 1.56$)$ \\
Left arm & $1.11(0.89$ to 1.39$)$ & 0.18 & $1.22(0.90$ to 1.65$)$ \\
Trunk & $1.16(0.93$ to 1.45$)$ & $\mathbf{0 . 0 4}$ & $1.10(0.94$ to 1.28$)$ \\
Right leg & $1.15(1.01$ to 1.31$)$ & $\mathbf{0 . 0 1}$ & $1.41(1.08$ to 1.83$)$ \\
Left leg & $1.27(1.05$ to 1.54$)$ & $\mathbf{0 . 0 3}$ & $1.30(1.01$ to 1.66$)$ \\
Whole body & $1.24(1.03$ to 1.51$)$ & $\mathbf{0 . 0 4}$ & $1.40(1.02$ to 1.91$)$ \\
\hline
\end{tabular}

${ }^{a}$ The multivariate logistic regression model included age, BMI: gender, education, income/year, hyperlipidemia, hypertension, diabetes, smoking, passive smoking, drinking, and the phase angle at a frequency of $50 \mathrm{kHz} . P$ values of $<0.05$ were considered statistically significant.

TABLE 4: The associations between CAP and phase angles at a frequency of $50 \mathrm{kHz}$.

\begin{tabular}{lcccr}
\hline \multirow{2}{*}{ Phase angles } & \multicolumn{2}{c}{$\beta(95 \% \mathrm{CI})$} & \multicolumn{2}{c}{ OR (95\% CI) } \\
& Univariate analysis & Multivariate analyses ${ }^{\mathrm{a}}$ & Univariate analysis & Multivariate analyses ${ }^{\mathrm{b}}$ \\
\hline Right arm & $6.11(1.17 \text { to } 11.06)^{*}$ & $1.40(-4.42$ to 7.23$)$ & $1.38(1.13 \text { to } 1.7)^{* *}$ & $1.09(0.83$ to 1.43$)$ \\
Left arm & $7.24(2.32 \text { to } 12.16)^{* *}$ & $2.09(-3.67$ to 7.85$)$ & $1.47(1.20 \text { to } 1.81)^{* * *}$ & $1.21(0.92$ to 1.58$)$ \\
Trunk & $5.20(2.45 \text { to } 7.94)^{* * *}$ & $2.93(-0.09$ to 5.95$)$ & $1.27(1.13 \text { to } 1.44)^{* * *}$ & $1.13(0.98$ to 1.31$)$ \\
Right leg & $8.72(4.51 \text { to } 12.93)^{* * *}$ & $9.69(4.79 \text { to } 14.59)^{* * *}$ & $1.46(1.22 \text { to } 1.74)^{* * *}$ & $1.45(1.15 \text { to } 1.82)^{* *}$ \\
Left leg & $8.86(4.53 \text { to } 13.19)^{* * *}$ & $9.40(4.33 \text { to } 14.46)^{* * *}$ & $1.47(1.23 \text { to } 1.76)^{* * *}$ & $1.43(1.13 \text { to } 1.81)^{* *}$ \\
Whole body & $9.27(4.37 \text { to } 14.17)^{* * *}$ & $7.20(1.32 \text { to } 13.09)^{*}$ & $1.54(1.25 \text { to } 1.89)^{* * *}$ & $1.34(1.02 \text { to } 1.76)^{*}$ \\
\hline
\end{tabular}

a,b Adjusted for age, BMI, gender, education, income/year, hyperlipidemia, hypertension, diabetes, smoking, passive smoking, and drinking. ${ }^{*}, P<0.05$; ${ }^{* *}, P<0.01 ; * * *, P<0.001$

Besides, logistic regression analyses were also used to estimate the associations between CAP and PhAs with a cut-off at $240 \mathrm{~dB} / \mathrm{min}$ for CAP. The results suggested that CAP were also significantly associated with the PhAs of the right leg, left leg and whole body (for right leg, OR = 1.45, 95\% CI: 1.15 to 1.82 ; for left leg, $\mathrm{OR}=1.43,95 \% \mathrm{CI}$ : 1.13 to 1.81 ; for whole body, $\mathrm{OR}=1.34,95 \% \mathrm{CI}: 1.02$ to 1.76 ; all $P<0.05)$.

The stratified analyses by BMI are shown in Table 5 . In the multivariate logistic regression models, there was no $\mathrm{PhA}$ associated with NAFLD and abnormal CAP in the participants with $\mathrm{BMI} \geq 30$. While in the participants with $\mathrm{BMI}<30$, the associations of the PhAs of the right leg, left leg, and whole body with NAFLD were significant (for the right leg, $\mathrm{OR}=1.70,95 \% \mathrm{CI}: 1.30$ to 2.22 ; for the left leg, $\mathrm{OR}=1.53$, 95\% CI: 1.17 to 2.00; for the whole body, OR $=1.65,95 \% \mathrm{CI}$ : 1.20 to 2.28; all $P<0.05$ ). Besides, the PhAs of the right leg, left leg, and whole body were also associated with abnormal CAP (for the right leg, $\mathrm{OR}=1.58,95 \% \mathrm{CI}$ : 1.22 to 2.05 ; for the left leg, $\mathrm{OR}=1.49,95 \% \mathrm{CI}: 1.15$ to 1.93 ; for the whole body, $\mathrm{OR}=1.47,95 \% \mathrm{CI}: 1.08$ to 2.00 ; all $P<0.05)$. 
TABLE 5: Multivariate logistic regression analyses of phase angles at a frequency of $50 \mathrm{kHz}$ with NAFLD and abnormal CAP, stratified analyses by BMI.

\begin{tabular}{lccrl}
\hline \multirow{2}{*}{ Phase angles } & \multicolumn{2}{c}{ NAFLD: OR $(95 \% \mathrm{CI})^{\mathrm{a}}$} & \multicolumn{2}{c}{$\begin{array}{c}\text { Abnormal CAP: OR (95\% CI })^{\mathrm{b}} \\
\text { BMI }<30\end{array}$} \\
\hline Right arm & $1.23(0.89$ to 1.68$)$ & $0.57(0.19$ to 1.75$)$ & $1.12(0.83$ to 1.52$)$ & $1.01(0.55$ to 1.85$)$ \\
Left arm & $1.33(0.97$ to 1.82$)$ & $0.68(0.24$ to 1.98$)$ & $1.29(0.96$ to 1.75$)$ & $1.07(0.59$ to 1.94$)$ \\
Trunk & $1.18(1.01$ to 1.39$)$ & $1.04(0.55$ to 1.97$)$ & $1.22(1.04 \text { to } 1.43)^{*}$ & $1.02(0.71$ to 1.46$)$ \\
Right leg & $1.70(1.30 \text { to } 2.22)^{* * *}$ & $0.66(0.27$ to 1.60$)$ & $1.58(1.22 \text { to } 2.05)^{* * *}$ & $1.39(0.82$ to 2.35$)$ \\
Left leg & $1.53(1.17 \text { to } 2.0)^{* *}$ & $0.63(0.25$ to 1.61$)$ & $1.49(1.15 \text { to } 1.93)^{* *}$ & $1.61(0.92$ to 2.79$)$ \\
Whole body & $1.65(1.20 \text { to } 2.28)^{* *}$ & $0.58(0.19$ to 1.76$)$ & $1.47(1.08 \text { to } 2.00)^{*}$ & $1.20(0.65$ to 2.24$)$ \\
\hline
\end{tabular}

a,b Adjusted for age, BMI, gender, education, income/year, hyperlipidemia, hypertension, diabetes, smoking, passive smoking, and drinking. ${ }^{*}, P<0.05$; ${ }^{* *}$, $P<0.01 ; * *, P<0.001$.

\section{Discussion}

In the present study, our findings showed that $\mathrm{PhAs}$ were significantly associated with the risk of NAFLD. The associations were still robust after further adjustment for potential confounders. Besides, we found significant associations between PhAs and CAP values, a common test for clinical diagnosis of NAFLD. The results suggested that PhA values might be effective indicators in the management of NAFLD among overweight people.

BIA has a lot of advantages, including noninvasive, maneuverable, objectivity, reproducibility, and low cost. BIA device can measure various body compositions, such as Lean Body Mass, body cell mass, Extracellular water, intracellular water, fat mass, fat free mass, and so on. In contrast to BIAderived body composition, i.e., fat mass and fat free mass, PhA does not depend on equations and their inherent assumptions [22]. BIA measures $\mathrm{PhA}$ through to bioelectricity parameters: body resistance $(R)$ and reactance $(\mathrm{Xc})$. PhA can be expressed as arctan $\mathrm{Xc} / R$. Resistance, the restriction of the flow of electrical current, is primarily related to the water in the tissues; reactance, the resistive effect produced by the tissue interfaces and cell membranes, can represent the cell membrane integrity [23]. Accumulating evidence has proposed that PhA could be a useful indicator for several clinical states, including cancer, cirrhosis, sarcopenia chronic obstructive pulmonary disease, and acute respiratory failure [24-27].

There was evidence that higher BMI was associated with increased $\mathrm{PhA}$ values because a higher BMI person tended to have more cells (fat or muscle cells) $[28,29]$. Lower PhA indicated decreased cell integrity and might be an accurate predictor of poor prognosis [11, 30]. Previous studies had confirmed that hepatocellular steatosis and excessive accumulation of extra-and intrahepatic fats was the hallmark of NAFLD. A study including 75 healthy adults and 48 NAFLD patients suggested that total body fat mass was significantly associated with the risk of NAFLD [31]. Our results were consistent with these reports. The findings that PhAs of the right leg, left leg, and whole body were significantly associated with the risk of NAFLD and CAP supports PhAs can be indicators to the prevention and management of NAFLD. However, the associations of the PhAs with NAFLD and abnormal CAP were only observed in the participants with $\mathrm{BMI}<30$, but not in the participants with $\mathrm{BMI} \geq 30$. In our study, the percentage of NAFLD was very high in the people with $\mathrm{BMI} \geq 30$, at about $93 \%$. Thus no significant associations were found in this group.

Our results indicated that significant associations were found in the PhAs of the right leg, left leg, and whole body, while the associations were not found in arms and truck PhA values. In the arm measurement with BIA, the high estimation error may occur due to the small tissue interface and cell number in arms. A previous report compared BIA with dual-energy X-ray absorptiometry in young wrestlers, showing that there was no high correlation of body composition in the arm [32]. As for the trunk, PhA values of trunk showed the lowest correlation with other parts in our study (Table S1). The liquid- and air-filled spaces of trunk can have an impact on the resistivity. Compared with limbs, the trunk has a much larger cross-sectional area, which may also cause its surface resistance to be very small. Nevertheless, muscle fibers of the truck are multidirectional and the current needs to pass through many different tissue interfaces, resulting in an increase of trunk resistance [33]. Thus, the trunk PhA is affected by these factors in the measurement.

To our knowledge, this is the first study attempting to establish a relationship between the PhA and NAFLD in an overweight population. In this study, we found that higher PhA was significantly associated with the risk of NAFLD. After controlling for confounders, the associations remained significant. There are some limitations to this study that should also be taken into account. First, the data were collected at a single care center. Then, it was unclear if we could extrapolate our findings to a normal weight population because our participants were all overweight. Further research is necessary to investigate the association between $\mathrm{PhA}$ and NAFLD in a normal weight population.

\section{Conclusion}

Our study has indicated that PhAs were significantly associated with the risk of NAFLD in an overweight population. Positive associations between $\mathrm{PhAs}$ and CAP values were also observed. The findings provide evidence that $\mathrm{PhAs}$ might be effective indicators in the prognosis of NAFLD in overweight people. Further investigations are needed to explore $\mathrm{PhA}$ in a larger population. 


\section{Data Availability}

Data are available upon request to the corresponding author.

\section{Conflicts of Interest}

The authors declare no conflicts of interest.

\section{Authors' Contributions}

Guangya Chen and Yi Lv contributed equally to this article.

\section{Acknowledgments}

This work was supported by grants from the National Natural Science Foundation of China (81703243 and 81573815).

\section{Supplementary Materials}

Table S1: correlations of phase angles among different body parts. (Supplementary Materials)

\section{References}

[1] M. Yi, R.-P. Chen, R. Yang, and H. Chen, "Increased prevalence and risk of nonalcoholic fatty liver disease in overweight and obese patients with type 2 diabetes in South China," Diabetic Medicine, vol. 34, no. 4, pp. 505-513, 2017.

[2] S. Zelber-Sagi, D. Shoham, I. Zvibel, S. Abu-Abeid, O. Shibolet, and S. Fishman, "Predictors for advanced fibrosis in morbidly obese nonalcoholic fatty liver patients," World Journal of Hepatology, vol. 9, no. 2, pp. 91-98, 2017.

[3] E. H. Van den Berg, M. Amini, T. C. Schreuder et al., "Prevalence and determinants of nonalcoholic fatty liver disease in lifelines: a large Dutch population cohort," PLOS One, vol. 12, Article ID e0171502, 2017.

[4] J. Yan, W. Xie, W. N. Ou et al., "Epidemiological survey and risk factor analysis of fatty liver disease of adult residents, Beijing, China," Journal of Gastroenterology and Hepatology, vol. 28, no. 10, pp. 1654-1659, 2013.

[5] X. Y. Zhao, X. Xiong, T. Liu et al., "Long noncoding RNA licensing of obesity-linked hepatic lipogenesis and NAFLD pathogenesis," Nature Communications, vol. 9, no. 1, pp. 1-14, 2018.

[6] K. Stavropoulos, K. Imprialos, A. Pittaras, C. Faselis, P. Narayan, and P. Kokkinos, "Lifestyle modifications in nonalcoholic fatty liver disease and nonalcoholic steatohepatitis," Current Vascular Pharmacology, vol. 16, no. 3, pp. 239-245, 2018.

[7] Q. Zhang, C. K. H. Wong, K. Kung et al., "Development and validation study of a nonalcoholic fatty liver disease risk scoring model among adults in China," Family Practice, vol. 34, no. 6, pp. 667-672, 2017.

[8] L. Li, D.-W. Liu, H.-Y. Yan, Z.-Y. Wang, S.-H. Zhao, and B. Wang, "Obesity is an independent risk factor for nonalcoholic fatty liver disease: evidence from a meta-analysis of 21 cohort studies," Obesity Reviews, vol. 17, no. 6, pp. 510-519, 2016.

[9] D. L. Dumitrascu and M. G. Neuman, "Nonalcoholic fatty liver disease: an update on diagnosis," Medicine and Pharmacy Reports, vol. 91, no. 2, pp. 147-150, 2018.
[10] I. Cova, S. Pomati, L. Maggiore et al., "Nutritional status and body composition by bioelectrical impedance vector analysis: a cross sectional study in mild cognitive impairment and Alzheimer's disease," PLoS One, vol. 12, Article ID e0171331, 2017.

[11] G. Vassilev, T. Hasenberg, J. Krammer, P. Kienle, U. Ronellenfitsch, and M. Otto, "The phase angle of the bioelectrical impedance analysis as predictor of post-bariatric weight loss outcome," Obesity Surgery, vol. 27, no. 3, pp. 665-669, 2017.

[12] R. Younes and E. Bugianesi, "A spotlight on pathogenesis, interactions and novel therapeutic options in NAFLD," $\mathrm{Na}$ ture Reviews Gastroenterology \& Hepatology, vol. 16, no. 2, pp. 80-82, 2019.

[13] A. Schwenk, A. Beisenherz, K. Römer, G. Kremer, B. Salzberger, and M. Elia, "Phase angle from bioelectrical impedance analysis remains an independent predictive marker in HIV-infected patients in the era of highly active antiretroviral treatment," The American Journal of Clinical Nutrition, vol. 72, no. 2, pp. 496-501, 2000.

[14] U. G. Kyle, L. Genton, and C. Pichard, "Low phase angle determined by bioelectrical impedance analysis is associated with malnutrition and nutritional risk at hospital admission," Clinical Nutrition, vol. 32, no. 2, pp. 294-299, 2013.

[15] F. Jian-gao and Chinese Liver Disease Association, "Guidelines for management of nonalcoholic fatty liver disease: an updated and revised edition," Zhonghua Gan Zang Bing Za Zhi, vol. 18, no. 3, pp. 163-166, 2010.

[16] S. Somda, A. Lebrun, H. Tranchart et al., "Adaptation of controlled attenuation parameter (CAP) measurement depth in morbidly obese patients addressed for bariatric surgery," PLoS One, vol. 14, Article ID e0217093, 2019.

[17] K. Pu, Y. Wang, S. Bai et al., "Diagnostic accuracy of controlled attenuation parameter (CAP) as a non-invasive test for steatosis in suspected nonalcoholic fatty liver disease: a systematic review and meta-analysis," BMC Gastroenterol, vol. 19, p. 51, 2019.

[18] M. Tobari, E. Hashimoto, M. Taniai et al., "Characteristics of nonalcoholic steatohepatitis among lean patients in Japan: not uncommon and not always benign," Journal of Gastroenterology and Hepatology, vol. 34, no. 8, pp. 1404-1410, 2019.

[19] P. Hartmann and B. Schnabl, "Risk factors for progression of and treatment options for NAFLD in children," Clinical Liver Disease, vol. 11, no. 1, pp. 11-15, 2018.

[20] X. E. Peng, S. H. Xu, W. Liu, Z. Hu, Z. Lin, and X. Lin, "Independent and combined effects of dietary iron composition and selected risk factors on the risk of NAFLD in a Chinese population," Scientific Reports, vol. 9, no. 1, p. 4069, 2019.

[21] M. J. Lee, E.-H. Kim, S.-J. Bae et al., “Age-related decrease in skeletal muscle mass is an independent risk factor for incident nonalcoholic fatty liver disease: a 10-year retrospective cohort study," Gut and Liver, vol. 13, no. 1, pp. 67-76, 2019.

[22] L. Genton, F. R. Herrmann, A. Sporri, and C. E. Graf, "Association of mortality and phase angle measured by different bioelectrical impedance analysis (BIA) devices," Clinical Nutrition, vol. 37, no. 3, pp. 1066-1069, 2018.

[23] M. S. Wladysiuk, R. Mlak, K. Morshed, W. Surtel, A. Brzozowska, and T. Malecka-Massalska, "Bioelectrical impedance phase angle as a prognostic indicator of survival in head-and-neck cancer," Current Oncology, vol. 23, no. 5, p. e481, 2016.

[24] K. Norman, N. Stobäus, D. Zocher et al., "Cutoff percentiles of bioelectrical phase angle predict functionality, quality of life, 
and mortality in patients with cancer," The American Journal of Clinical Nutrition, vol. 92, no. 3, pp. 612-619, 2010.

[25] C. Faisy, A. Rabbat, B. Kouchakji, and J.-P. Laaban, "Bioelectrical impedance analysis in estimating nutritional status and outcome of patients with chronic obstructive pulmonary disease and acute respiratory failure," Intensive Care Medicine, vol. 26, no. 5, pp. 518-525, 2000.

[26] G. Belarmino, M. C. Gonzalez, R. S. Torrinhas et al., "Phase angle obtained by bioelectrical impedance analysis independently predicts mortality in patients with cirrhosis," World Journal of Hepatology, vol. 9, no. 7, pp. 401-408, 2017.

[27] M. K. Kilic, M. C. Kizilarslanoglu, G. Arik et al., "Association of bioelectrical impedance analysis-derived phase angle and sarcopenia in older adults," Nutrition in Clinical Practice, vol. 32, no. 1, pp. 103-109, 2017.

[28] M. C. G. Barbosa-Silva, A. J. Barros, J. Wang, S. B. Heymsfield, and R. N. Pierson, "Bioelectrical impedance analysis: population reference values for phase angle by age and sex," The American Journal of Clinical Nutrition, vol. 82, no. 1, pp. 49-52, 2005.

[29] A. Bosy-Westphal, S. Danielzik, R.-P. Dörhöfer, W. Later, S. Wiese, and M. J. Müller, "Phase angle from bioelectrical impedance analysis: population reference values by age, sex, and body mass index," Journal of Parenteral and Enteral Nutrition, vol. 30, no. 4, pp. 309-316, 2006.

[30] D. A. Pérez Camargo, S. R. Allende Pérez, M. M. Rivera Franco, N. E. Álvarez Licona, V. I. Urbalejo Ceniceros, and L. E. Figueroa Baldenegro, "Phase Angle of bioelectrical impedance analysis as prognostic factor in palliative care patients at the National Cancer Institute in Mexico," Nutrition and Cancer, vol. 69, no. 4, pp. 601-606, 2017.

[31] M. Balakrishnan, H. B. El-Serag, T. Nguyen, J. Hilal, F. Kanwal, and A. P. Thrift, "Obesity and risk of nonalcoholic fatty liver disease: a comparison of bioelectrical impedance analysis and conventionally-derived anthropometric measures," Clinical Gastroenterology and Hepatology, vol. 15, no. 12, pp. 1965-1967, 2017.

[32] M.-f. Cheng, Y.-Y. Chen, T.-R. Jang, W.-L. Lin, J. Chen, and K.-C. Hsieh, "Total body composition estimated by standingposture 8-electrode bioelectrical impedance analysis in male wrestlers," Biology of Sport, vol. 33, no. 4, pp. 399-405, 2016.

[33] R. N. Baumgartner, W. C. Chumlea, and A. F. Roche, "Estimation of body composition from bioelectric impedance of body segments," The American Journal of Clinical Nutrition, vol. 50, no. 2, pp. 221-226, 1989. 